\title{
Blockchain Network as a Platform: Conceptualising its Adapted Layered Architecture Design
}

Runyu Shi

Warwick Business School, University of Warwick, UK

Correspondence: mandyshiry@gmail.com

Received: 15 January 2021 Accepted: 18 April 2021 Published: 6 May 2021

\begin{abstract}
Digital platform as an organising logic has prominently reshaped the innovation activities in many sectors. Previous studies have extensively investigated the digital platforms from two views: economic view (i.e. as a sided marketplace) and engineering view (i.e. as an innovation infrastructure). Blockchain - a digital artefact that connects the distributed ledgers - resembles great overlaps and specifics to digital platforms. Building on this aspect, I first demarcate the Blockchain Product as a Platform (BPaaP) informed by the economic view and the Blockchain network as a platform $(\mathrm{BNaaP})$ inspired by the engineering view. Given the scant of research around $\mathrm{BNaaP}$, this research aims to depict the BNaaP's architectural design by drawing on the layered design of digital technologies. Using Ping An Group as a case, this research applies the thematic analysis method in analysing online open data. As the main contribution, this research proposes the Adapted Layered Architecture of BNaaP that consists of (1) three design layers (foundation layer, interaction layer, and application layer); (2) adapted business scenarios; and (3) environmental factors. The suggested architectural design implies the BNaaP's internal synergistic collaboration among layers and the external adaptability to different business contexts. Overall, this research provides a novel angle to understand the Blockchain phenomenon and brings insightful implications to Blockchain practitioners.
\end{abstract}

Keywords: digital platforms; Blockchain platforms; Blockchain network; case study; thematic analysis

JEL Classifications: M15

\section{Introduction}

Digital platforms that enable the value co-creation of multiple parties that are otherwise not connected have prominently reshaped our economy and daily life [1] [2]. Blockchain, as an emerging digital solution, resembles great similarities and distinctiveness to digital platforms. Specifically, past studies on digital platforms have well established two salient views: platform as a marketplace (i.e. economic view) [3] [4] and platform as an innovation infrastructure (i.e. engineering view) [5] [6]. The economic view emphasises the platform's effectiveness in enabling transactions among multiple parties that are otherwise disconnected. Blockchain echoes this view through its collaborative nature of the distributed ledgers and its subject to the network effects. Alternatively, the engineering view discusses how a platform can incorporate heterogeneous innovation while maintaining unity [7]. In this regard, establishing a Blockchain platform requires synergistic co-creation within its design structure and entails high adaptability to different contextual scenarios.

Informed by the economic view and engineering view are two possible pathways of studying Blockchain. The first strand considers Blockchain as a marketplace that enables value exchange among multiple parties. Very often, participants in the Blockchain market have a certain degree of a joint agreement on Blockchain product's value. This article defines this stream as Blockchain Product as a Platform (BPaaP). Examples include cryptocurrencies such as Bitcoin, Facebook's Libra, digital tokens, and the Initial Coin Offering (ICO). Platform participants are bounded by the common recognition of the digital product's exchange value and the collective objective to continually grow the value of Blockchain product.

The other stream proposes that the Blockchain's usefulness is not embedded in its transaction value but more emerged from its architectural design as an organisation logic. Shedding light on Blockchain's enablement of building and sustaining a network, this article defines this type of Blockchain as Blockchain Network as a Platform (BNaaP). Instead of focusing on digital asset transactions, $\mathrm{BNaaP}$ grants more efforts in articulating the overarching design of the Blockchain network. In other words, despite what products are transmitted through Blockchain, BNaaP emphasises on how Blockchain platform is established with multiple levels of complexity and is adapted to 
different business scenarios where platform participants may have diverse value exchange expectations.

Motivated by the importance of $\mathrm{BNaaP}$ as an organisation logic, this article aims to establish the fundamental ground of the architecture design of $\mathrm{BNaaP}$. The case understudied is Ping An Group's Oneconnect Blockchain solution. I use the thematic analysis method to analyse online data and obtain some humanistic insights on BNaaP. The proposed Adapted Layered Architecture serves as a starting point that inspires future research on $\mathrm{BNaaP}$ specifics. It also provides insightful implications to practitioners who wish to utilise Blockchain to ignite business transformation.

\section{Related literature}

\subsection{Digital platforms}

The platform has been widely recognised and applied as an organisation structure that enables multiple parties to exchange value and incorporate external parties' innovation [8]. The different sides of participants, governance rules and regulations, and various platform resources form the platform ecosystem [1] [9]. Prior studies on platforms have been mainly built on two streams. The first stream is founded on the market nature of platforms. This economic view considers the platform as a mediator that facilitates transactions among multiple types of otherwise not connected users. Such user connection tradition is widely revealed by the two-sided or multi-sided organisation of platforms [3]. Catalysed by such sided-platform organisation is the network effects, which indicate the reinforced traction of growing one side of the platform users (e.g. app users) in attracting the other side (e.g. app developers). The platform's usefulness to each user is most likely to increase as more people join from the same side or the other side [4] [10].

Another popular stream of platform research is built on the engineering view. Unlike the economic tradition that highlights the transactional feature, the engineering view discusses how platforms can be a resource pool that facilitates external parties' innovation activities [6] [1]. In this regard, platforms often consist of a core product, on top of which are a set of platform resources (e.g. Application Programming Interfaces, Software Development Kits, and development guidelines) that aim to enable innovation from heterogenous developers [11]. The arm-length distance between platform owners and platform participants enables platforms to grow in generativity that extends the platform core product's usefulness. For example, to augment the Android operating system's generativity, Google has issued many platform resources to different participants such as the Google APIs and SDKs to developers, the hardware-abstraction layer, compatibility test suite, and MADA (mobile application distribution agreement) to Android device manufacturers, and some other user tools such as Android studio. In this regard, a platform can be viewed as a digital infrastructure that fulfils the core requirements of its intended users and entails flexibilities to be modified, updated, and adapted [12].
Digital platforms, as a design hierarchy, are greatly dependent on and enabled by the capabilities of digital technologies. Yoo, Henfridsson, and Lyytinen [13] suggest that different from the integral design hierarchy, which entails closely binded interdependency among different components and in between the product's functionality and its physical embodiment, the digital technologies are featured by reprogrammability and datahomogeneity. First, the reprogrammability implies the possible separation of the technology's functional logic and its physical embodiment. As long as the users agree on the digital resource's general meaning, they have much freedom to decide how they would like these resources to produce and perform. Users can combine different types of digital recourses to serve their user cases. Alternatively, they may re-engineer the usefulness of digital assets by modifying source codes. Second, the datahomogeneity ensures the consistency of digital data across devices and networks. It means digital data that was installed and altered on one device can be seamlessly synchronised in multiple places. The data-homogeneity enables the wider transmission of digital information and ensures the unrestcited user innovation on multiple devices.

The objective of exploring the Blockchain platform's design architecture brings the engineering view more relevant to this study. Inspired by Yoo et al. [13], this research will depict the Blockchain platforms' architecture design by drawing on the layered architecture design of digital technology. The layered architecture lays the foundation to view the Blockchain network as an integrated digital artefact formed of multiple functioning layers [14]. Inside each layer are many sub-systems and modules that correspond to different types of utilities.

\subsection{Blockchain platforms}

Since the debut of Bitcoin, Blockchain technology has gradually influenced business activities in many different sectors such as finance, manufacture, and public management. Widely known as a database formed by the distributed ledgers, Blockchain in nature resembles several common attributes to platforms regarding its features of connecting isolated entities and facilitating digital exchange [15]. However, Blockchain also exhibits many features that make it go beyond the scope of a sided-platform.

First, as a distributed ledger organisation, Blockchain presents the dissolved boundary of the same-side and cross-side network effects. Participants who contribute a new block to the existing chain are also users who benefit from the chain's increased usefulness. Because the ledger records all types of participants' activities (e.g. add transactions and modify information) on the network and publish these records to each participant [16], adding a new member or a new block to the chain network always implies a higher degree of information richness to each node [16]. More conservatively speaking, though some cross-side leverage relationship may exist (e.g. block contributor and block beneficiary), its significance on a Blockchain platform is much less than that on a typical sided platform. 
Second, different from digital platforms where governance rules and practices are mostly performed by a central party, that is, platform owners, Blockchain allows decentralised governance [17]. The modification and alteration of Blockchain must pass the authentication of all members on the chain. There is no single entity that can enforce the dominant power to decide what can or cannot be done [18]. Besides, because participants in a chain network have a common agreement on disclosing information in return for a collective efficiency, a single entity can rarely manipulate the information asymmetry and make the entire Blockchain to privilege its own business. Such distributed governance and eliminated central dominance highlight the Blockchain platform's distinct differences from the conventional digital platforms.

Third, following decentralised governance, another benefit realised by Blockchain is digital trust. All data shared on the Blockchain network is encrypted. Members' rights to read and modify can be classified with classified deciphering keys. Therefore, participants, in general, have fewer concerns in hiding or manipulating the data integrity. Also, with the full records of the chain activities, the Blockchain entails high traceability which means that any intentional sabotage of chain integrity can be traced to its sources.

Upon establishing the overlaps and different features presented by digital platforms and Blockchain platforms, this article has introduced two meaningful Blockchain pathways: $\mathrm{BPaaP}$ and $\mathrm{BNaaP}$. The $\mathrm{BPaaP}$ relies on the transaction nature of Blockchain platforms; the BNaaP offers an early emphasis on the value of Blockchain architecture as an organising logic. With ample empirical examples around BPaaP (e.g. Bitcoin and Facebook's Libra), this research aims to shed light on the unique value of $\mathrm{BNaaP}$. To offer some fundamental groundings to this research stream, I will investigate the architectural design of $\mathrm{BNaaP}$. The depicted framework aims to open new venues for future research and offer blueprint guidance to practitioners.

\section{Methodology}

To investigate the architecture design of the $\mathrm{BNaaP}$, this research will conduct a case study. As an explorative research, the data used in this research mainly consists of internet-based qualitative data. The thematic analysis method will be applied to gain insights from online data.

It is noted that the case study "is not a methodological choice but a choice of what is to be studied" [19]. Given the early spread of Blockchain and the scant empirical evidence, doing a case study has substantial usefulness to acquire new experiences, humanistic understanding, and knowledge about this theme [20] [21]. In this regard, an in-depth exploration of the single case helps understand the Blockchain architecture's complexity and particularity [22] [23]. The case selected in this study aims to constitute a representative member of larger organisation groups [24]. And the knowledge gained from this single case is deemed to provide naturalistic generalisability [20].
This research follows Braun and Clarke's [25] thematic analysis method to analyse online qualitative data. By definition, the thematic analysis "is a method for identifying, analysing, and reporting patterns (themes) within data" [25]. In other words, it offers the possibility to capture the meaning of data by identifying different themes and their associations from the data. Adopting the thematic analysis method fulfils the objective of this study in the following two ways:

First, the thematic analysis does not hold a committed subscription to the development of a theory. According to Braun and Clarke [25], for an under-researched area, rather than theory building, the thematic analysis can capture predominant and important themes in relation to the available dataset. Applying this method may sacrifice the depth of analysis, but the overall description and articulation will be well captured and maintained [25] [23]. Second, the thematic analysis entails some allowance for the researchers to preconceive a certain degree of coding direction informed by other theories. This feature demarcates thematic analysis from the grounded theory, which often implies the delayed literature engagement [26]. In this research, some insights from the platform literature essentially help to sensitise themes and interpret the Blockchain network architecture. Therefore, applying thematic analysis with wide references to platform studies well fulfils the research objectives.

\section{Data and analysis}

Blockchain technology has received rapid development in the past five years in China. In line with digital technology's prominence, the National Strategic Development department strongly emphasises the Blockchain's vital influence on the future economy. To highlight, the National Plan for Information Technology during the Period of the Thirteenth Five-Year Plan (2016-2020) remarked Blockchain as a frontier weapon for the new wave of national development and competition. Over the years, some national authorities and relevant institutions gradually issued a series of documents, guidance, regulations, and industry standards to incentivise Blockchain innovation.

The case studied in this research is Ping An Insurance (Group) Company of China, Ltd. Established in 1988, it is one of the largest financial service providers in mainland China and ranked 29th in Fortune Global 500 in 2019. As a financial institution with a highly diversified business portfolio, Ping An Group has always adhered to the "finance + technology + ecosystem" principle. In recent years, Ping An Group has extensively innovated with different digital technologies. It revolutionized the bank operations using Artificial Intelligence technologies that helped to build an open banking system and connect bank users, clients, and business partners. This AI banking intelligently managed business operations and monitored risks at a larger scale. Besides, Ping An Group also launched its OneConnect subsidiary. With 161 patents, the OneConnect's Blockchain solution has seen successful deployment in many business sectors such as finance, cross-border trade, estates, automobile, healthcare, and smart-city. The OneConnect's 
Blockchain solution is designed as an infrastructure that facilitates business activities on a network basis, thus serving as a valuable empirical case to this study.

Guided by the thematic analysis method, this study primarily obtained data from Ping An Group's online official publications. The dataset includes (a) 12 annual and semiannual reports from 2014 to 2019 published by Ping An Group - these documents precisely reveal the firm's trajectory of digital advancement over the years; (b) 27 newsletters and 4 "white page" documents published by Ping An's OneConnect Research Institution - these documents provide detailed insights, particularly on the development, deployment, and regulations around the Blockchain application; (c) multiple media data sources, including 7 online interviews and public speech clips from Ping An's Co-CEO and Chief Innovation Officer; and (d) 32 articles published by media and research organisations such as Bloomberg, OECD, China Academy of Information and Communications Technology, and International Data Corporation (IDC). These documents supplement an alternative angle to assess the Blockchain's application in different industries and its business impacts.

The thematic analysis of the collected data is applied with the six-step analysis suggested by Braun and Clarke [25]. First, media data in video forms are transcribed into scripts. All collected data are cleaned and saved into Nvivo software and are read twice by the researcher to gain initial sensitisation. Second, each document is analysed with open coding strategies. Nodes are coded to data blocks whenever they are implying "basic segment, or element of the raw data or information that can be assessed in a meaningful way regarding the phenomenon" [25]. This step results in 45 first-level nodes and 29 sub-level nodes. Third, the coded nodes obtained in the second step are reviewed and sorted into 14 potential themes, which can best capture coded data's core meaning. Up to this stage, a thematic map draft is made with relationships and associations of all themes. Before moving on to the theme refinement, two graduate students are involved as research assistants to read all documents independently and review all the nodes. Fourth, the researcher revisits all coded data and the proposed themes. This stage mainly investigates the internal homogeneity within each theme and the external heterogeneity among themes [24]. Besides, this stage also engages the evaluation of the theme's "accurate representation" of the entire dataset [25]. Fifth, each theme's essence and the thematic map are re-assessed with several iterations of data review. In the last step, the thematic map is finalised. Themes are defined, interpreted, and discussed with data extracts.

\section{Results}

Figure 1 presents the proposed architectural design of BNaaP. The term "layered" describes how the Blockchain platform is developed and managed. The term "adapted" implies its applicability and adaptability when implemented in different business scenarios. In the following, I will explain each conceptualisation construct with empirical evidence.

\subsection{Foundation layer}

The foundation layer describes the technical specifications that are necessary for establishing a Blockchain platform. Three subordinate layers are included. First, the technological layer deals with hardware and software solutions that substantially enable Blockchain functionalities. For example, the requestresponse module deals with the ongoing tension between the block size and processing transactions' speed. A larger block size enables high throughput. However, when transaction volume is low, such a large block design often leads to high latency. Ping An's "smart block" solution adopts the blockless structure, which can achieve almost real-time responses without reducing transaction throughput.

Second, the functional layer involves digital solutions that aim to ensure the integrity, authenticity, and accountability of chain functionality and operation. For instance, in the cryptography module, Ping An patents its 3D zeroknowledge proof technology, which can verify data and statements within three milliseconds without revealing the real information enclosed in the block. Besides, at the functional layer, the management of digital keys is also a critical task in sustaining Blockchain operation. It determines whether the Blockchain can maintain its operation quality with a high level of security.

Third, the operational layer deals with Blockchain's usefulness and effectiveness in supporting different user needs. This layer consists of some deployment tools and management tools. For example, OneConect's Blockchain invented many primary and add-on business-specific functionalities for a different category of users. Specifically, it enables customised asset record format, allows asset liquidation based on users' changing status, and monitors abnormalities with the user-defined Web interfaces. Compared to the technological and functional layers, the operational layer focuses more on the business operation aspect and the user-interface design.

\subsection{Interaction layer}

While the foundation layer ensures the well-functioning of the Blockchain, the interaction layer embodies the possibility of forming a Blockchain platform using some boundary resources. Three types of boundary resources are involved. The general APIs allow a chain to quickly incorporate some commonly used functionalities such as identity management and digital contract. The industry APIs are groups of interfaces that are designed to fit the needs of a specific industry. It allows some industry-level services to be incorporated instantaneously. For example, in the international trading context, such APIs may involve orders, invoices, and logistics. The third interactive element is the cross-chain APIs. This type of APIs is widely engaged when a Blockchain network wants to connect with an external Blockchain network. Overall, the foundation layer ensures the usefulness of the interaction layer. And the interaction layer extends the capabilities and scalability of the foundation layer. 


\subsection{Application layer}

The application layer describes the possible means and complexity when deploying a Blockchain network. Companies and consumers can decide to join an existing Blockchain network by instantaneously attaching themselves to the Blockchain nodes. Alternatively, they can operationalise the foundation layer and the interactive layer to initiate a new Blockchain network. Depending on the business needs, joining an existing Blockchain network will benefit the joined party with pre-existed network value. Because the incumbent Blockchain network has already acquired a large number of relevant business partners, built relatively mature procedures, and tested its operational efficiency and security standards. Nevertheless, creating a new Blockchain network may be necessary if no existing chain fits the business requirements. Moreover, enterprises may also consider building a new Blockchain platform as a means of leveraging and reengineering the network value of their business circles.

\subsection{Adapted business scenario}

Conceptualising the $\mathrm{BNaaP}$ architecture is not completely done if we omit the varying implementation business scenarios. The business scenarios indicate the purposes and contexts of the Blockchain network. Because the types of business activities, the potential capacity of the network, and the required standards and regulations will all affect the operation and management of the Blockchain platform. For example, Ping An Group helped Hong Kong Monetary Authority to establish an eTradeConnect Blockchain platform. This platform involved 12 international banks from the Hong Kong region and an independent trading platform (we.Trade) in Europe. The Blockchain platform's global orientation required Ping An to ensure that all designs at the foundation and interaction layer were complying with both parties' legal obligations. However, in the other case, Ping An's Blockchain platform was used by a Chinese local government to achieve faster document transmission and communication with those connected organisations. In such a context, the Blockchain's compliance to international legal obligations became less relevant. The Blockchain's flexibility in adapting to users with different levels of technical knowhow and distinct habits of sharing documents became a central challenge.

\subsection{Environmental factors}

The environmental factor is a critical element that influences the establishment and growth of Blockchain platforms. This factor contains multiple parties that are either directly or indirectly engaged in the Blockchain development. Informed by the Ping An's Blockchain network, environmental factors' impacts can be grouped into four categories: (1) Monitor. While encrypted data is only readable for the trading parties on the chain, the national monitoring authority may have full access to view all the trading information. (2) Regulate. The Cyberspace Administration of China has issued a series of
Blockchain-relevant regularities that have enforcement power to all Blockchain applications. (3) Arbitrate. On Ping An Blockchain, signing an electronic contract is under the supervision of the National Notarization and Judicial Departments. If there is any dispute, the notary department will intervene. (4) Certificate. The data security suite adopted by Ping An Blockchain has obtained the National Certification of Level 3 Information Security Protection provided by the Ministry of Public Security.

Besides, it is worth noting that the development and growth of the Blockchain platform also recursively feedbacks fresh insights into the environmental factors' alteration and improvement. For instance, Ping An's 3D zero-knowledge proof technology and its crypto-controlled data sharing technology significantly accelerate the modification of Blockchain security regulations at the industrial level.

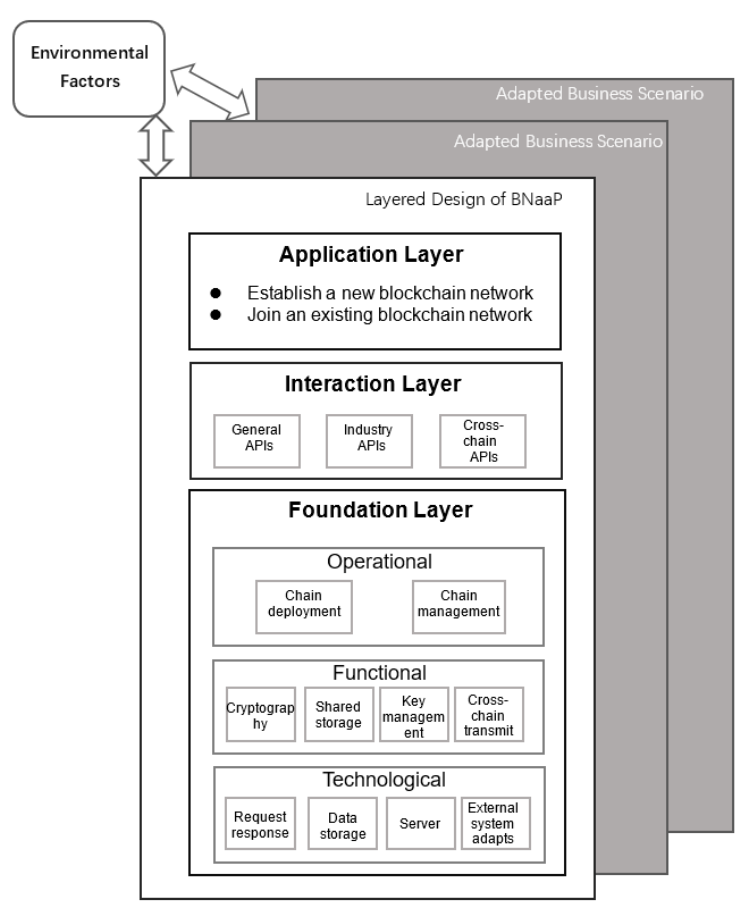

Figure 1: The Adapted Layered Architecture of BNaaP.

\section{Conclusion}

Inspired by the similarities and differences of digital platforms and Blockchain network, this study, for the first time, suggests two potential pathways of viewing and studying Blockchain phenomenon: $\mathrm{BPaaP}$ and $\mathrm{BNaaP}$. Given the scant attention to the $\mathrm{BNaaP}$, this research aims to arouse interests towards this stream by investigating its architectural design. The proposed Adapted Layered Architecture of BNaaP depicts a blueprint to practitioners who wish to leverage the power of Blockchain in igniting digital business transformation. Besides, it also serves as a starting point for future research to explore the formation and implementation of $\mathrm{BNaaP}$ from multiple aspects, including but not limited to the technical challenges, governance issues, business adaption considerations, and the bilateral relationship with environmental factors. 
However, this research also presents some limitations. First, the data used in this research contains online open data published by the understudied case company. The officiality of these materials ensures data authentication and validity. Future improvement may involve an attempt to gain more first-party insights from interviews. Second, the aims of gaining knowledge on the overarching design of $\mathrm{BNaaP}$ neglect the importance and complexity of the "adapting" processes. Future studies can approach this aspect by adopting the process view to study Blockchain's adaption tactics and specifics in relation to different business factors.

\section{Competing Interests:}

None declared..

\section{Ethical approval:}

Not applicable.

\section{Author's contribution:}

Runyu Shi is the main author responsible for writing the manuscript, collecting data, proofreading, etc.

\section{Funding:}

Not applicable.

\section{Acknowledgements:}

Not applicable.

\section{References:}

[1] M. G. Jockbides, A. Sundararajan and M. Van Alstyne, "Platforms and Ecosystems: Enabling the Digital Economy," World Economic Forum, Switzerland, 2019.

[2] A. Tiwana, B. Konsynski and A. Bush, "Platform Evolution: Coevolution of Platform Architecture, Governance, and Environmental Dynamics," Information Systems Research, vol. 21, no. 4, pp. 675-687, 2010.

[3] J. Rochet and J. Tirole, "Two-Sided Markets: A Progress Report.," RAND Journal of Economics, vol. 35, pp. 645-667, 2006.

[4] M. Armstrong, "Competition in Two-Sided Markets," RAND Journal of Economics, vol. 37, pp. 668-691, 2006.

[5] A. Gawer, "Bridging Differing Perspective on Technological Platforms: Toward an Integrative Framework," Research Policy, vol. 43, pp. 1239-1249, 2014.

[6] K. Clark, "The Interaction of Design Hierarchies and Market Concepts in Technological Evolution," Research Policy, vol. 14, no. 5, pp. 235-251, 1985.

[7] K. Karhu, R. Gustafsson, B. Eaton, O. Henfridsson and S. Carsten, "Four Tactics for Implementing a Balanced Digital Platform Strategy," MIS Quarterly Executive, vol. 19, no. 2, pp. 105-120, 2020.

[8] G. Parker, M. Van Alstyne and X. Jiang, "Platform Ecosystesm: How Developers Invert The Firm," MIS Quarterly, vol. 44, no. 1, pp. 255-266, 2017.
[9] A. Tiwana, "Platform Synergy: Architectural Origines and Competitive Consequences," Information Systems Research, vol. 29, no. 4, pp. 829-848, 2018.

[10] M. Rysman, "The Economics of Two-Sided Markets," Journal of Economic Perspectives, vol. 23, no. 3, pp. 125-143, 2009.

[11] M. Meyer and A. Lehnerd, "The Power of Product Platforms: Building Value and Cost Leadership," New York: Free Press, 1997.

[12] S. Wheelwright and K. Clark, "Creating Project Plans to Focus Product Development," Harvard Business Review, vol. 70, no. 2, pp. 67-83, 1992.

[13] Y. Yoo, O. Henfridsson and K. Lyytinen, "The New Organising Logic of Digital Innovation: An Agenda for Information Systems Research," Information Systems Research, vol. 21, no. 4, pp. 724-735, 2010.

[14] D. Teece, "Economics of Scope and the Scope of the Enterprise," Behavior and Organization, vol. 1, pp. 223-247, 1980.

[15] Z. Zheng, S. Xie, H. Dai, X. Chen and H. Wang, "An Overview of Blockchain Technology: Architecture, Concensus, and Future Trends," Honolulu, USA, 2017.

[16] M. Nofer, P. Gomber and O. Hinz, "Blockchain," Business \& Information Systems Engineering, vol. 59, pp. 183-187, 2017.

[17] R. van Pelt, S. Jansen, D. Baars and S. Overbeek, "Defining Blockchain Governance: A Framework for Analysis and Comparison," Information Systems Management, vol. 38, no. 1, pp. 21-41, 2021.

[18] R. Beck, C. Muller-Bloch and J. King, "Governance in the Blockchain Economy: A Framework and Reseach Agenda," Journal of the Association for Information Systems, vol. 19, no. 10, p. Article 1, 2018.

[19] R. Stake, "Qualitative Case Studies," in The Sage Handbook of Qualitative Research, Thousand Oaks, CA: Sage, 2006, pp. 433-466.

[20] R. Stake, "The Case Study Method in Social Inquiry," Educational Researcher, pp. 5-8, 1978.

[21] R. Boyatzis, "Transforming Qualitative Information: Thematic Analysis and Code Development," Thousand Oaks, CA: Sage, 1998

[22] R. Stake, "The Art of Case Research," Thousand Oaks, CA: Sage, 1995.

[23] H. Simons, "Case Study Research in Practice," SAGE Publications, 2009.

[24] M. Patton, "Qualitative Evaluation and Research Methods (2nd ed.)," Newbury Park, CA: Sage, 1990.

[25] V. Braun and V. Clarke, "Using Thematic Analysis in Psychology," Qualitative Research in Psychology, vol. 3, no. 2, pp. 77-101, 2008.

[26] Urquhart, "Grounded Theory for Qualitative Research," SAGE Publications, 2013. 